\title{
Phonon Scattering in Silicon by Multiple Morphological Defects: A Multiscale Analysis
}

\author{
BRUNO LORENZI ${ }^{1}$ RICCARDO DETTORI,${ }^{2}$ MARC T. DUNHAM, ${ }^{3}$ \\ CLAUDIO MELIS, ${ }^{2,4}$ RITA TONINI, ${ }^{5}$ LUCIANO COLOMBO, ${ }^{2,4}$ \\ ADITYA SOOD,${ }^{3,6}$ KENNETH E. GOODSON,${ }^{3}$ and DARIO NARDUCCI (1) ${ }^{1,7}$ \\ 1.-Department of Materials Science, University of Milano-Bicocca, via Cozzi 55, 20125 Milan, \\ Italy. 2.-Department of Physics, University of Cagliari, Cittadella Universitaria, \\ 09042 Monserrato, CA, Italy. 3.-Department of Mechanical Engineering, Stanford University, \\ Stanford, CA 94305, USA. 4.-Istituto Officina dei Materiali, CNR-IOM SLACS Cagliari, \\ Cittadella Universitaria, 09042 Monserrato, CA, Italy. 5.-Department of FIM, University of \\ Modena and Reggio Emilia, via Campi 213, 41100 Modena, Italy. 6.-Department of Materials \\ Science and Engineering, Stanford University, Stanford, CA 94305, USA. 7.-e-mail: \\ dario.narducci@unimib.it
}

Ideal thermoelectric materials should possess low thermal conductivity $\kappa$ along with high electrical conductivity $\sigma$. Thus, strategies are needed to impede the propagation of phonons mostly responsible for thermal conduction while only marginally affecting charge carrier diffusion. Defect engineering may provide tools to fulfill this aim, provided that one can achieve an adequate understanding of the role played by multiple morphological defects in scattering thermal energy carriers. In this paper, we study how various morphological defects such as grain boundaries and dispersed nanovoids reduce the thermal conductivity of silicon. A blended approach has been adopted, using data from both simulations and experiments in order to cover a wide range of defect densities. We show that the co-presence of morphological defects with different characteristic scattering length scales is effective in reducing the thermal conductivity. We also point out that non-gray models (i.e. models with spectral resolution) are required to improve the accuracy of predictive models explaining the dependence of $\kappa$ on the density of morphological defects. Finally, the application of spectral models to Matthiessen's rule is critically addressed with the aim of arriving at a compact model of phonon scattering in highly defective materials showing that non-local descriptors would be needed to account for lattice distortion due to nanometric voids.

Key words: Thermal conductivity, thermoelectricity, silicon, phonons

\section{INTRODUCTION}

The thermal conductivity $\kappa$ of a crystalline material depends upon several factors, including point defects, ${ }^{1}$ surface scattering, ${ }^{2}$ and isotopic distribution. ${ }^{3}$ Thermal conductivity may be also significantly reduced by the introduction of morphological defects such as dislocations, grain boundaries, precipitates, pores, and other extended $(1 \mathrm{D}, 2 \mathrm{D}$, and

(Received January 22, 2018; accepted April 26, 2018;

published online May 11, 2018)
3D) defects. In the development of high-performance thermoelectric materials, it is of paramount importance to optimize the efficiency of scatterers*, that, in principle, should be selected so as to decrease $\kappa$ while marginally impacting the electrical conductivity $\sigma$. This has been shown to be feasible in several instances. Possibly the most dramatic display was provided in the case of silicon nanowires, ${ }^{4,5}$ where a proper choice of nanowire wall roughness

*Here we deal with materials where heat carriers are mainly phonons. 
led to a more than tenfold decrease of $\kappa$ while retaining $\sigma$ values close to those of bulk singlecrystalline silicon. It is therefore not surprising that over the last few years, a large number of papers have appeared proposing more sophisticated theoretical approaches to the analysis of phonon scattering. A key question they addressed was regarding the contributions of optical and acoustic phonons to the macroscopic thermal conductivity, and how they were suppressed by morphological defects.

In lithographically defined single-crystalline silicon membranes, it was shown that the introduction of cylindrical pores with diameters ranging from 1.9 to $10.9 \mu \mathrm{m}$ leads to a reduction of $\kappa$ depending on the pore diameter up to room temperature. ${ }^{6}$ This is a non-trivial observation if one considers that in the gray model (i.e. using a single, effective phonon frequency to describe the contribution of all phonon modes), the phonon mean free path ( $\mathrm{mfp})$ at room temperature in bulk silicon is around $0.2 \mu \mathrm{m} .{ }^{7}$ A recent experiment carried out in $p$-type polycrystalline silicon films ${ }^{8}$ reported a decrease of $\kappa$ with the hole radius, ranging from $120 \mathrm{~nm}$ down to 30 $\mathrm{nm}$, in periodic structures with a fixed period of 300 $\mathrm{nm}$. Grain sizes were widely distributed, extending from $10 \mathrm{~nm}$ to $100 \mathrm{~nm}$, thus overlapping with hole radii. Analysis led to the conclusion that thermal conductivity was controlled by volume reduction, sensibly through the heat capacity. However, a more detailed evaluation of the scattering mechanisms was not possible.

The analysis of the reduction of thermal conductivity due to grain boundaries (GBs) and porosity also motivated a revision of the standard kinetic model. Dames et al. ${ }^{9}$ showed that below the Debye temperature $\Theta_{\mathrm{D}}$, the thermal conductivity of nanocrystalline silicon pellets obtained by spark plasma sintering followed a quadratic (rather than cubic) dependence on temperature. This suggested an $\omega$-dependent mfp, with $\Lambda \propto \omega^{-1}$. Hua and Minnich $^{10}$ refined the model using Monte Carlo simulations, and confirmed a frequency-dependent phonon transmissivity at grain boundaries. Simulations showed that up to $\approx 60 \%$ of the total heat is carried by phonons with mfp longer than the grain size. A similar temperature dependence at $T<\Theta_{\mathrm{D}}$ was also reported in porous silicon. Silicon inverse opals $^{11}$ displayed $\kappa \sim T^{1.8}$ while mesoporous nanocrystalline silicon thin films ${ }^{12}$ reported $\kappa \sim T^{2}$. In the former case, the low-temperature $\kappa(T)$ was explained by invoking a quadratic frequency dependence of the scattering rate at grain boundaries due to coherent phonon reflection, while in mesoporous silicon, the quadratic increase of $\kappa$ with $T$ was explained using the minimum thermal conductivity model derived by Cahill and Pohl in 1988 for amorphous or strongly defective crystals. ${ }^{13}$ No frequency-dependent transmissivity at pore surfaces was proposed.
The impact of the $\omega$ dependence on the mfp at room temperature or above is still unclear in polycrystalline materials. According to Wang et al. $^{9}$ the spectral dependence of the $\mathrm{mfp}$ is of practical relevance with respect to the thermal conductivity only at low temperatures. On the other hand, Hua and Minnich ${ }^{10}$ as well as Jiang et al. ${ }^{14}$ have noted the importance of accounting for the frequency dependence of the $\mathrm{mfp}$ over the whole temperature range.

This state of affairs provides evidence that there is still an open issue about the best physically sound model to describe the effect of morphological defects on the mean free path of microscopic heat carriers. The question is whether it is better to treat the full phonon spectrum through effective concepts or, rather, if it is necessary to treat each single mode individually, since defects operate differently at different frequencies. ${ }^{15,16}$ Hereafter we will refer to the first kind of model as gray, while addressing each mode independently depending on its frequency as non-gray. This issue also impacts the actual usability of the adopted model. Simplified, effective models characterized by ease of implementation and by a reduced computational workload may enable computational analyses of materials of high structural complexity. On the contrary, a rigorous model treating each frequency mode independently may be practically applicable only to smaller systems, disallowing structurally accurate simulations of micromorphologically complex solids.

This paper addresses the usability of the gray model to model phonon scattering in defective solids around and above room temperature by exploring the dependence of the thermal conductivity on the size and density of morphological defects (MoDs). First, single-crystalline and polycrystalline silicon films, both porous and non-porous, were simulated to compute $\kappa$. Second, thermal conductivity was experimentally measured for polycrystalline silicon films which were $\mathrm{He}^{+}$-implanted and thermally processed to promote the formation of pores. ${ }^{17} \mathrm{By}$ keeping the characteristic length scale of grains approximately fixed in both the computational and experimental investigations, we were able to isolate the effects of the pores on phonon filtering.

We chose silicon as the material in which to study these effects due to its well-characterized intrinsic thermal properties, which have been extensively studied both experimentally ${ }^{18-21}$ and computationally $^{22}$ in thin films and in bulk single crystals. ${ }^{23}$ Additional motivation stems from the renewed interest for thermoelectric applications of silicon and Si-based systems using either bulk materials $^{24-26}$ or thin films. ${ }^{27-30}$ It should be remarked, however, that nanovoids have also been shown to positively impact the thermoelectric properties of other materials. Decrease of thermal conductivity was reported in porous filled skutterudites ${ }^{31}$ and, very recently, in unfilled skutterudites as well. ${ }^{32}$ 


\section{PHONON SCATTERING IN MULTI-SIZED DEFECTIVE CRYSTALS}

\section{Background}

The standard kinetic model relates the lattice thermal conductivity at temperature $T$ to phonon mean free path $\Lambda(\omega, s, T)$ and velocity $v(\omega, s)$, both quantities depending on phonon frequency $\omega$ and on polarization $s$ :

$$
\kappa(T)=\frac{1}{3} \sum_{s} \int_{0}^{\infty} C(\omega, s, T) v(\omega, s) \Lambda(\omega, s, T) \mathrm{d} \omega
$$

where $C(\omega, s, T)$ is the spectral specific heat of the material and the sum runs over all polarizations. In addition to phonon-phonon and phonon-electron scattering, the phonon mfp is limited by the presence of defects, which have different scattering capabilities depending on $\omega$ and on a typical length scale $\ell$ associated with the defect itself. In single crystals, phonons are uniquely labeled by their wavevector $q$ and by their polarization $s$, namely $\omega=\omega(q, s)$. For the thermal conductivity to be described in the $\mathrm{mfp}$ (direct) space, it was suggested $^{7}$ that the mfp $\Lambda$ of a phonon might also be labeled using the same four scalars, i.e. $\Lambda=\Lambda(q, s)$. Under the (widely verified) assumption that both $\omega=\omega(q, s)$ and $\Lambda=\Lambda(q, s)$ be at least locally monotonic functions of $q_{x}, q_{y}$, and $q_{z}$, one may then invert $\Lambda(q, s)$ so that $\omega=\omega(\Lambda, s)$. Thus, an mfp spectral function of the thermal conductivity may be defined

$$
K(\Lambda, T)=-\sum_{s} \frac{1}{3} C(\Lambda, s, T) v(\Lambda, s, T) \Lambda\left(\frac{\mathrm{d} \Lambda}{\mathrm{d} \omega}\right)^{-1}
$$

where both $C(\Lambda, s, T)$ and $v(\Lambda, s, T)$ are here written as a function of the mfp. Thus

$$
\kappa(T)=\int_{0}^{\infty} K(\Lambda, T) \mathrm{d} \Lambda
$$

The normalized thermal conductivity accumulation function $\alpha(\Lambda, T)$ is further defined as

$$
\alpha\left(\Lambda_{\alpha}, T\right)=\frac{1}{\kappa} \int_{0}^{\Lambda_{\alpha}} K(\Lambda, T) \mathrm{d} \Lambda
$$

In the presence of a single $\mathrm{MoD}$, thermal conductivity scales as

$$
\kappa_{\mathrm{MoD}}(T)=\int_{0}^{\infty} K_{\mathrm{b}}\left(\Lambda_{\mathrm{b}}, T\right) B_{\mathrm{b}}^{\mathrm{MoD}}\left(\Lambda_{\mathrm{b}}, \ell_{\mathrm{MoD}}\right) \mathrm{d} \Lambda_{\mathrm{b}}
$$

where ' $\mathrm{b}$ ' refers to the MoD-free material and $B_{\mathrm{b}}^{\mathrm{MoD}}\left(\Lambda_{\mathrm{b}}, \ell_{\mathrm{MoD}}\right) \equiv \Lambda_{\mathrm{MoD}} / \Lambda_{\mathrm{b}}$ is some function of the ratio $\Lambda_{\mathrm{b}} / \ell_{\mathrm{MoD}}$ that depends on the type of defect.

In the construction of the $B$ term, an important role is played by the way different scatterers contribute to set the mfp. Although $B$ is in no way constrained to any specific rule of $\mathrm{mfp}$ combination, in practice, the use of Matthiessen's rule has almost no alternative, in spite of the increasing number of papers noting its limits. ${ }^{33-35}$ Matthiessen's rule assumes that in the presence of several scattering events, each characterized by a scattering time $\tau_{i}$, an effective scattering time $\tau$ may be defined as

$$
\tau^{-1}=\sum_{i} \tau_{i}^{-1}
$$

or, equivalently, it is assumed that there is no interplay among scattering mechanisms. Thus,

$$
\Lambda=\frac{1}{\sum_{i} \Lambda_{i}^{-1}}
$$

We will refer to this implementation of Matthiessen's rule as the integral Matthiessen's rule. As known, its proposal moves from the gray hypothesis, namely that a dominant phonon mode of frequency $\omega_{\mathrm{G}}$ exists, so that Eq. 1 reduces to its simplified kinetic form $\kappa=1 / 3 c v \Lambda_{\mathrm{G}}$, where $c$ is the (integral) specific heat at constant volume. It is instead well known (Ref. 36, p. $307)$ that phonon modes are not independent, so that scattering events mix up modes both by normal and Umklapp processes.

An alternate way to estimate the $\mathrm{mfp}$ in the presence of several scattering phenomena is to use the spectral Matthiessen's rule. An effective specific scattering time $\tau(\omega, s)$ is defined for each phonon mode of frequency $\omega$ and polarization $s$ as

$$
\tau(\omega, s)^{-1}=\sum_{i} \tau_{i}(\omega, s)^{-1}
$$

(where $\tau_{i}(\omega, s)$ is the scattering time for phonon modes of frequency $\omega$ and polarization $s$ due to the $i$ th scattering event) so that, since $\Lambda_{i}(\omega, s)=v(\omega, s) \tau_{i}(\omega, s)$,

$$
\Lambda(\omega, s)=\frac{v(\omega, s)}{\sum_{i} \tau_{i}(\omega, s)^{-1}}=\frac{1}{\sum_{i} \Lambda_{i}(\omega, s)^{-1}}
$$

It should be noted that not even the spectral Matthiessen's rule provides a rigorous way to compute the effective mfp $\Lambda(\omega, s)$ as it also neglects mode mixing. Nonetheless, it does not rely upon the dominant mode assumption, so that it may be more properly and consistently used when non-gray mfp models are introduced.

\section{Spectral Functions}

In what follows, we will make use of two $\mathrm{mfp}$ spectral functions $K_{\mathrm{b}}\left(\Lambda_{\mathrm{b}}, T\right)$. For the gray model, we will consistently assume that

$$
K\left(\Lambda_{\mathrm{b}}\right)=\kappa_{\mathrm{b}} \delta\left(\Lambda_{\mathrm{b}}-\Lambda_{\mathrm{b}, \mathrm{G}}\right)
$$

with $\Lambda_{\mathrm{b}, \mathrm{G}}=205 \mathrm{~nm}$ at room temperature for silicon. ${ }^{7}$ In the gray model, $\Lambda_{\mathrm{b}, \mathrm{G}}$ is the $\omega$-independent mfp of phonons that is set by scattering from extended defects (Ref. 36, p. 316). For the non-gray models, we will use instead the $\mathrm{mfp}$ spectral 
function obtained by complementing computational data by Esfarjani et al. ${ }^{37}$ and Jiang et al. ${ }^{14}$

\section{COMPUTATIONAL PROCEDURE}

\section{Sample Preparation}

In this work, using computer simulations, we generated three different prototypical silicon structures, namely: (a) samples containing both a random and an ordered distribution of pores, but no GBs; (b) samples containing a GB network as well as a random distribution of pores; and (c) samples where pores are pinned at GBs. The generation of such a library of samples required two distinct procedures for pores and GBs, respectively.

The pores are created by randomly selecting a suitable number of lattice sites and removing all atoms within a distance $d_{\mathrm{p}}$. This generates pores and since they are randomly distributed, the probability of creating a pore is adjusted to achieve a target porosity $\phi_{\mathrm{p}}$, defined as

$$
\phi_{\mathrm{p}} \equiv \Omega_{\mathrm{p}} / \Omega
$$

where $\Omega_{\mathrm{p}}$ is the volume pertaining to the pores while $\Omega$ is the medium volume. Further iterations are carried out until the desired porosity is obtained within a certain tolerance. As the simulation protocol requires a uniform mass distribution along the transport direction (see the following subsection), the generation of pores is similarly applied within sequential regions of the sample, so as to obtain a uniform porosity. Following this procedure, samples with suitable pore density have been generated, with diameter ranging from $0.47 \mathrm{~nm}$ to $2.6 \mathrm{~nm}$, resulting in an overall porosity of $\phi_{\mathrm{p}}=0.005$. A similar procedure has been applied to obtain ordered pore distributions, with the only difference that the pore seeds were placed according to a cubic lattice. In this case, two porosity values $\phi_{\mathrm{p}}=0.028$ and $\phi_{\mathrm{p}}=0.005$ have been considered. All samples required an accurate structural optimization protocol. The potential energy was at first minimized by means of conjugate gradient optimization. Next, a simulated annealing process was applied, heating the samples up to $900 \mathrm{~K}$ and cooling them back to room temperature for a total simulation time of $900 \mathrm{ps}$, with a final equilibration time of $400 \mathrm{ps}$ at $300 \mathrm{~K}$. Further information about this procedure can be found elsewhere. ${ }^{38}$

The generation of GBs required an ad hoc multistep computational protocol: a crystalline sample with dimensions $L_{x}=2.72 \mathrm{~nm}, L_{y}=27.15 \mathrm{~nm}$ and $L_{z}=135.76 \mathrm{~nm}$ (containing as many as $5 \times 10^{5}$ atoms) was fully amorphized following a standard quenching-from-the-melt approach. Then, $N_{\mathrm{g}}$ sites were selected at random in the $y z$ plane and around each of them a cylindrical region (passing across the full $L_{x}$ dimension of the simulation box) was created in order to replace the amorphous matter with a crystalline cylinder, randomly rotated in the $y z$ plane.
The resulting structures (amorphous matrices decorated by crystalline seeds) were annealed at constant $T=1200 \mathrm{~K}$ for $3.0 \mathrm{~ns}$. In this way, seed grains could grow until a nanocrystalline silicon system was eventually created. The number $N_{\mathrm{g}}$ of initial seeds was chosen in order to fill the $y z$ plane with grains of average size $D=25.0 \mathrm{~nm}$. A detailed description of this protocol is reported in Refs. 39 and 40.

By combining the above two procedures, a number of systems were obtained, differing in atomicscale nanostructure while keeping the same holey polycrystalline morphology, as shown in Fig. 1.

A third kind of holey polycrystalline sample was realized, where pores were pinned at GBs. For this purpose, pore generation was slightly modified. It is known that atoms in an amorphous structure are characterized by a higher configurational energy, with respect to atoms in a crystalline lattice. According to the environment-dependent interaction potential (EDIP) adopted in this work, ${ }^{41}$ the average energy for a crystalline silicon atom is $\left\langle E_{\mathrm{c}-\mathrm{Si}}\right\rangle=-4.6 \mathrm{eV}$; thus, a further requirement for the generation of pores was to involve only atoms with energy greater than $\left\langle E_{\mathrm{c}-\mathrm{Si}}\right\rangle$. We remark that this interaction scheme has been adopted since it is accurate in describing non-crystalline forms of silicon, as well as in predicting the thermal transport properties of disordered silicon forms, e.g., amorphous and nanocrystalline ones. ${ }^{38,40,42,43}$

Holey polycrystalline samples of categories (b) and (c) are characterized by porosity values $\phi_{\mathrm{p}}=0.005$, with $0.47 \geq d_{\mathrm{p}} \geq 2.6 \mathrm{~nm}$.

\section{Computational Details}

Thermal conductivity $\kappa$ was calculated at room temperature in periodically repeated simulation cells during the system's approach to equilibrium, proceeding from an initial nonequilibrium configuration where the two similar portions of the simulation cell were set at temperature $T_{1}=400 \mathrm{~K}$ and $T_{2}=200 \mathrm{~K}$, respectively. By computing on the fly

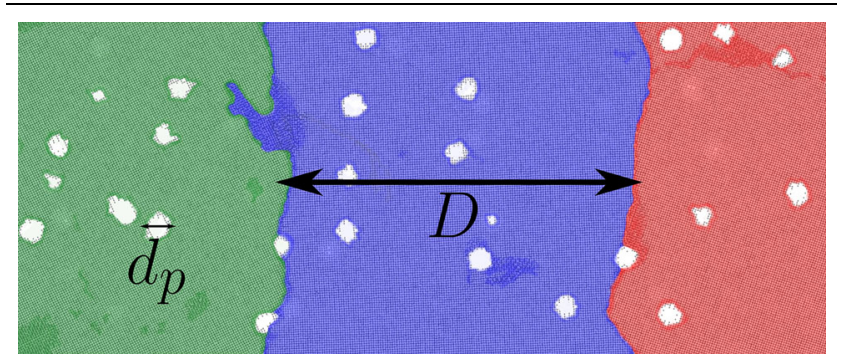

Fig. 1. (color online) Illustration of the atomic-scale structure of a holey polycrystalline sample with randomly distributed pores. Different colors represent differently oriented crystalline grains; darker areas identify defect-rich pockets, e.g. amorphous spots or grain boundaries. Two relevant morphological features are discussed in the paper, $d_{p}$ and $D$, are shown. The picture shows a 0.9-nm-thick longitudinal section, corresponding to just $\approx 1 / 3$ of the overall sample thickness. We remark that the simulation cell is as thick as $2.72 \mathrm{~nm}$ and, therefore, matter is in fact not discontinuous, although some atoms seem to be unbound. 
the evolving temperature difference $T(t)=\left\langle T_{1}(t)\right\rangle-$ $\left\langle T_{2}(t)\right\rangle$ (where $\langle\ldots\rangle$ indicates the average separately taken in the two portions), we determined $\kappa$ from Ref. 42

$$
\Delta T(t)=\left\langle T_{1}\right\rangle-\left\langle T_{2}\right\rangle=\sum_{j=1}^{\infty} s_{j} e^{-\alpha_{j}^{2} t \kappa / c}
$$

The coefficients

$$
s_{j}=8\left(T_{1}-T_{2}\right) \frac{\left[\cos \left(\alpha_{j} L_{z} / 2\right)-1\right]^{2}}{\alpha_{j}^{2} L_{z}^{2}}
$$

and $\alpha_{j}=2 j / L_{z}$ are obtained for a system of length $L_{z}$ initially prepared in the configuration described above. An extensive explanation of this method, its validation, and several successful applications to Sibased systems can be found in Ref. 42 .

The equations of motion have been integrated by the velocity Verlet algorithm with time steps as short as 2 fs. Simulations were performed using the LAMMPS $^{44}$ package. The typical approach-to-equilibrium run lasted for as many as $3 \times 10^{5}$ timesteps, thus allowing a careful fit of the calculated $\Delta T(t)$ by Eq. 12.

Since present thermal conductivity data for nanocrystalline porous samples are calculated for finite-sized simulation cells, the actual normalizing value $\kappa_{\mathrm{sc}}$ corresponding to pristine silicon was evaluated for a system with the same length along the direction of thermal transport as nanocrystalline porous ones, similarly to previous investigations. ${ }^{45,46}$ Since this length in the present case was as short as $162.92 \mathrm{~nm}$, the corresponding conductivity of pristine silicon was set to $45.4 \pm 0.2 \mathrm{~W} \mathrm{~m}^{-1} \mathrm{~K}^{-1}$, as obtained by the present simulations.

\section{RESULTS}

In this section we will systematically analyze the thermal conductivity of silicon upon introduction of one or more morphological defects. Expressions for $\kappa$ in the gray and non-gray models will be obtained and compared to experimental data and/or to numerical simulations. This will enable an evaluation of the suitability of the gray approximation and of Matthiessen's rule, either spectral or integral, to explain the dependence of the thermal conductivity upon the characteristic defect size. We will show as well how in an already defective material further introduction of MoDs affects the thermal conductivity only when typical MoD sizes differ widely from each other.

\section{Single Morphological Defects in Silicon}

\section{Polycrystals}

It is quite natural to assume that GBs in polycrystalline materials limit the phonon $\mathrm{mfp}$ to the grain size $D$ so that $\Lambda_{\mathrm{P}}^{-1} \equiv \Lambda_{\mathrm{b}}^{-1}+\Lambda_{\mathrm{GB}}^{-1}=\Lambda_{\mathrm{b}}^{-1}+D^{-1}$. However, such an assumption is possibly too clear- cut. First, more than a single scattering event may be needed to fully thermalize phonons. Furthermore, phonons may be scattered with different efficiencies by grain boundaries depending on the misorientation of adjacent grains. Even in a gray model, grain boundaries may be assumed to transmit phonons with variable transmissivity $\mathcal{T}_{\mathrm{G}}$ depending upon the grain pair misorientation. Both factors suggest that we write

$$
\Lambda_{\mathrm{GB}}=\beta\left\langle\left(\frac{1}{\mathcal{T}_{\mathrm{G}}}-1\right)^{-1}\right\rangle D \equiv \beta \gamma_{\mathrm{G}} D
$$

with $\beta(\geq 1)$ counting the average number of scattering events needed by phonons to recover their equilibrium distribution, while $\gamma_{G}(\geq 1)$ scales the effective grain size by the grain boundary transmissivity. The use of $\beta$, which accounts for the difference between scattering and relaxation times, is reminiscent of the approach developed by Das Sarma and Stern for the analysis of electron scattering. ${ }^{47}$ Therefore, within the limits of validity of the integral Matthiessen's rule, the gray model leads to

$$
\Lambda_{\mathrm{P}, \mathrm{G}}^{-1}=\Lambda_{\mathrm{b}}^{-1}+\left(\beta \gamma_{\mathrm{G}} D\right)^{-1}
$$

where the subscript ' $\mathrm{P}$ ' labels the polycrystalline material. Thus, one gets

$$
B_{\mathrm{b}}^{\mathrm{P}, \mathrm{G}}=\left(1+\frac{\Lambda_{\mathrm{b}}}{\beta \gamma_{\mathrm{G}} D}\right)^{-1}
$$

In view of Eq. 5, this leads immediately to

$$
\begin{aligned}
\kappa_{\mathrm{P}, \mathrm{G}} & =\kappa_{\mathrm{b}} \int_{0}^{\infty} \delta\left(\Lambda_{\mathrm{b}}-\Lambda_{\mathrm{b}, \mathrm{G}}\right)\left(1+\frac{\Lambda_{\mathrm{b}}}{\beta \gamma_{\mathrm{G}} D}\right)^{-1} \mathrm{~d} \Lambda_{\mathrm{b}} \\
& =\kappa_{\mathrm{b}}\left(1+\frac{\Lambda_{\mathrm{b}, \mathrm{G}}}{\beta \gamma_{\mathrm{G}} D}\right)^{-1}
\end{aligned}
$$

which is the sought expression of the thermal conductivity in the gray approximation.

From an alternate perspective, following Wang et al. ${ }^{9}$ and Hua and Minnich, ${ }^{10}$ phonons may be transmitted by GBs with a transmissivity $\mathcal{T}(\omega)$

$$
\mathcal{T}(\omega)=\frac{1}{\gamma \omega / \omega_{\max }+1}
$$

where $\gamma$ is a fitting parameter and $\omega_{\max }$ is the maximum phonon frequency. Therefore, $\Lambda_{\mathrm{GB}}(\omega)=\gamma^{-1}\left(\omega_{\max } / \omega\right) \beta D$, namely GBs are transparent to phonons in the long-wavelength limit. Thus, the non-gray model predicts, still within the limits of validity of the spectral Matthiessen's rule, that

$$
\Lambda_{\mathrm{P}, \mathrm{NG}}^{-1}=\Lambda_{\mathrm{b}}^{-1}+(\gamma / \beta)\left(\omega / \omega_{\max }\right) D^{-1}
$$

where the subscript ' $\mathrm{NG}$ ' refers to the non-gray model.

The use of Eq. 19 is less straightforward as the mfp depends explicitly upon $\omega$. This implies that 


$$
B_{\mathrm{b}}^{\mathrm{P}, \mathrm{NG}}=\left(1+\frac{\gamma \omega}{\beta \omega_{\max }} \frac{\Lambda_{\mathrm{b}}}{D}\right)^{-1}
$$

For the most common bulk scattering mechanisms, ${ }^{48}$

$$
\Lambda_{\mathrm{b}}=A(T) \omega^{-n}
$$

where $n$ is a non-negative integer depending on the dominant scattering mechanism, and $A(T)$ is independent of $\omega$. In the whole numerical analysis carried out in this paper, phonon impurity scattering is assumed to dominate scattering in bulk (single-crystalline) silicon due to the high doping level. Independent of the phonon dispersion relation, it was shown ${ }^{37,48,49}$ that setting $n=4$ in Eq. 21 leads to a satisfactory description of the bulk mfp. Then, one may write

$$
B_{\mathrm{b}}^{\mathrm{P}, \mathrm{NG}}=\frac{\Lambda_{\mathrm{P}, \mathrm{NG}}}{\Lambda_{\mathrm{b}}}=\left[1+\frac{\gamma / \beta}{\omega_{\max }} \frac{\Lambda_{\mathrm{b}}}{D}\left(\frac{\Lambda_{\mathrm{b}}}{A}\right)^{-1 / 4}\right]^{-1}
$$

so that

$$
\kappa_{\mathrm{P}, \mathrm{NG}}=\int_{0}^{\infty} K_{\mathrm{b}}\left(\Lambda_{\mathrm{b}}\right)\left[1+\frac{\gamma / \beta}{\omega_{\max }} \frac{\Lambda_{\mathrm{b}}}{D}\left(\frac{\Lambda_{\mathrm{b}}}{A}\right)^{-1 / 4}\right]^{-1} \mathrm{~d} \Lambda_{\mathrm{b}}
$$

which states the dependence of the thermal conductivity in polycrystalline silicon is according to the non-gray model.

Figure 2 displays the fits** of Eqs. 17 and 23 to experimental data reported by Wang et al. ${ }^{9}$ for microcrystalline silicon and by Claudio et al. ${ }^{25}$ for nanocrystalline silicon. Both models are in good agreement with data for nanocrystalline silicon, although the non-gray model fits them more closely. Instead, only the non-gray model returns an acceptable fit for microcrystalline silicon. Setting $\omega_{\max }=12 \mathrm{THz}^{9,52}$ we get $A=(5.3 \pm 1.2) \times 10^{50} \mu \mathrm{m}$ $\mathrm{s}^{-4}$ for both sets of data, along with $\gamma / \beta$ values of $2.39 \pm 0.07$ and $0.28 \pm 0.02$, respectively.

\section{Holey single-crystalline silicon}

Holey semiconductors, namely single-crystalline semiconductors containing nanopores, have been widely studied both computationally and experimentally. A model for the mfp was proposed by some of the present authors. ${ }^{38}$ In short, the presence of

\footnotetext{
**Here and in the whole paper, fitting of the experimental or simulated data $\left\{\ldots,\left(x_{j}, y_{j}\right), \ldots\right\}$ to model equations $y=f\left(\ldots, a_{k}, \ldots ; x\right)$ was carried out using a Levenberg-Marquardt algorithm ${ }^{50}$ validated by simulated annealing, ${ }^{51}$ ensuring that the minimizer $\Psi\left(\ldots, a_{k}, \ldots\right)=\sum_{j}\left(f\left(\ldots, a_{k}, \ldots ; x_{j}\right)-y_{j}\right)^{2}$ had converged to an absolute minimum. Therefore, no equivalently accurate (or better) fitting (same or lower $\Psi$ values) may be factually achieved with a distinct set of fitting parameters $\left(\ldots, a_{k}, \ldots\right)$. Fitting sensitivity to each parameter is displayed by their standard deviations.
}

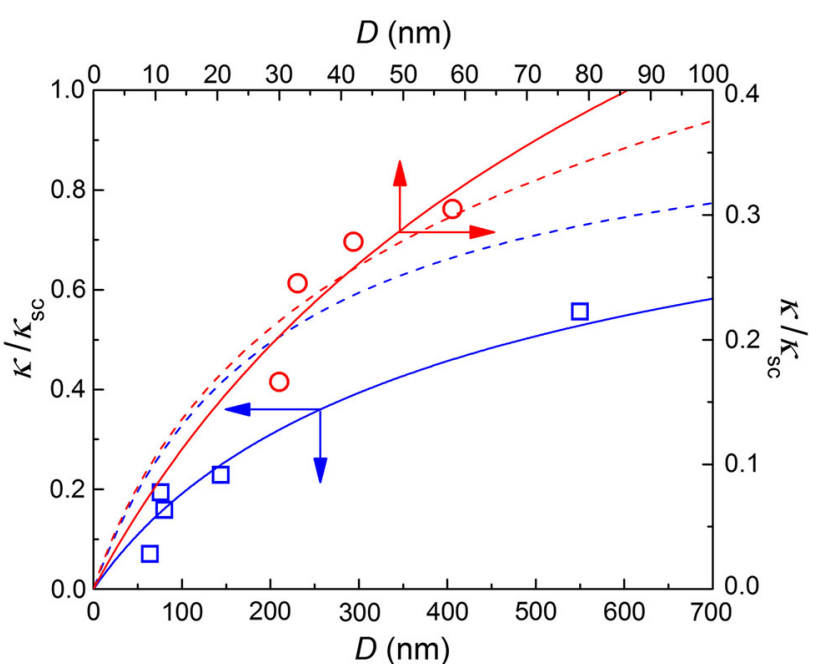

Fig. 2. (color online) Fitting of the normalized experimental values $\kappa / \kappa_{\text {sc }}$ versus grain size in polycrystalline silicon to the gray (dashed lines) and non-gray (full lines) models. Experimental data are from Wang et al. ${ }^{9}$ (square box, microcrystalline silicon) and from Claudio et al. ${ }^{25}$ (open circle, nanocrystalline silicon). Thermal conductivity $\kappa_{\mathrm{sc}}$ of single-crystalline silicon was, respectively, 142 and $87.3 \mathrm{~W} \mathrm{~m}^{-1}$ $\mathrm{K}^{-1}$.

single-sized pores with diameter $d_{\mathrm{p}}$ in a medium leads to two concurrent effects on the phonon $\mathrm{mfp}$ and and on the thermal conductivity. For any pore size, the effective medium approximation predicts, through the Maxwell-Garnett-Eucken (MGE) model, ${ }^{53}$ that

$$
\kappa_{\mathrm{H}, \mathrm{P}}=\kappa_{\mathrm{b}} \frac{1-\phi_{\mathrm{p}}}{1+\frac{\phi_{\mathrm{p}}}{2}}
$$

Bearing in mind that $C_{\mathrm{H}}(\omega, T)=C_{\mathrm{b}}(\omega, T)\left(1-\phi_{\mathrm{p}}\right)$ (where the subscript ' $\mathrm{H}$ ' labels holey systems), one may easily verify that the pertinent $\mathrm{mfp} \Lambda_{\mathrm{MGE}}$ relates to porosity and boundary $\mathrm{mfp}$ as

$$
1 / \Lambda_{\mathrm{MGE}}=\left(\phi_{\mathrm{p}} / 2\right)\left(1 / \Lambda_{\mathrm{b}}\right)
$$

This is only the effect of macroscopic pores and results from the reduction of the filled volume in the medium.

An additional component arises for micro-/nanopores, that relates instead to the density of scattering centers at the pore-solid interface. As reported in Ref. 38, the number of collisions per travelled distance $N_{\text {coll }}$ is given by the ratio between the projected pore cross section and the system volume. If $n_{\mathrm{p}}$ is the pore density, then

$$
N_{\text {coll }}=n_{\mathrm{p}} \pi\left(d_{\mathrm{p}} / 2\right)^{2}
$$

In view of Eq. 11, one may also write that

$$
\phi_{\mathrm{p}}=n_{\mathrm{p}} \frac{4 \pi}{3}\left(d_{\mathrm{p}} / 2\right)^{3}
$$

so that replacing $n_{\mathrm{p}}$ from the previous equation into Eq. 26, one gets 


$$
N_{\text {coll }}=\frac{3 \phi_{\mathrm{p}}}{2 d_{\mathrm{p}}}
$$

and the spacing between scattering centers reads

$$
\ell_{\mathrm{p}}=\frac{1}{N_{\text {coll }}}=\frac{2 d_{\mathrm{p}}}{3 \phi_{\mathrm{p}}}
$$

Thus, we may take the gray scattering length to sum up through the integral Matthiessen's rule, yielding

$$
1 / \Lambda_{\mathrm{H}, \mathrm{G}}=\left(\phi_{\mathrm{p}} / 2\right)\left(1 / \Lambda_{\mathrm{b}}\right)+1 /\left(\beta \ell_{\mathrm{p}}\right)
$$

where $\beta$ counts the number of scattering events needed to fully thermalize phonons. Therefore

$$
\frac{1}{\Lambda_{\mathrm{H}, \mathrm{G}}}=\frac{1}{\Lambda_{\mathrm{b}}}+\left(\frac{\phi_{\mathrm{p}}}{2 \Lambda_{\mathrm{b}}}+\frac{3 \phi_{\mathrm{p}}}{2 \beta d_{\mathrm{p}}}\right)
$$

and

$$
B_{\mathrm{b}}^{\mathrm{H}, \mathrm{G}}=\frac{\Lambda_{\mathrm{H}, \mathrm{G}}}{\Lambda_{\mathrm{b}}}=\left[1+\frac{\phi_{\mathrm{p}}}{2}\left(1+\frac{3 \Lambda_{\mathrm{b}}}{\beta d_{\mathrm{p}}}\right)\right]^{-1}
$$

so that finally

$$
\begin{gathered}
\kappa_{\mathrm{H}, \mathrm{G}}=\kappa_{\mathrm{b}} \int_{0}^{\infty} \delta\left(\Lambda_{\mathrm{b}}-\Lambda_{\mathrm{b}, \mathrm{G}}\right)\left[1+\frac{\phi_{\mathrm{p}}}{2}\left(1+\frac{3 \Lambda_{\mathrm{b}}}{\beta d_{\mathrm{p}}}\right)\right]^{-1} \mathrm{~d} \Lambda_{\mathrm{b}} \\
=\kappa_{\mathrm{b}}\left[1+\frac{\phi_{\mathrm{p}}}{2}\left(1+\frac{3 \Lambda_{\mathrm{b}, \mathrm{G}}}{\beta d_{\mathrm{p}}}\right)\right]^{-1}
\end{gathered}
$$

which is the expression for $\kappa$ in the presence of nanopores and in the gray approximation.

For the non-gray model, we need to account for the probability that phonons are elastically reflected at the pore surface. Actually, phonon transmissivity through a vacuum gap is exactly zero, while even at the nanoscale, the near-field thermal radiation contribution may be easily verified to be negligible. ${ }^{54}$ Using the so-called Ziman formula, ${ }^{36}$ reflectivity $R$ at pores reads

$$
R(\omega)=\exp \left(-\frac{16 \pi^{3} d_{\mathrm{p}}^{2} \omega^{2}}{v^{2}}\right)
$$

Thus,

$$
B_{\mathrm{b}}^{\mathrm{H}, \mathrm{NG}}=\frac{\Lambda_{\mathrm{H}, \mathrm{NG}}}{\Lambda_{\mathrm{b}}}=\left\{1+\frac{\phi_{\mathrm{p}}}{2}+\frac{3 \phi_{\mathrm{p}} \Lambda_{\mathrm{b}}}{2 \beta d_{\mathrm{p}}}[1-R(\omega)]\right\}^{-1}
$$

that, using again Eq. 21, leads to the explicit expression for $\kappa_{\mathrm{H}, \mathrm{NG}}$ :

$$
\begin{aligned}
& \kappa_{\mathrm{H}, \mathrm{NG}}=\int_{0}^{\infty} K_{\mathrm{b}}\left(\Lambda_{\mathrm{b}}\right)\left(1+\frac{\phi_{\mathrm{p}}}{2}+\frac{3 \phi_{\mathrm{p}} \Lambda_{\mathrm{b}}}{2 \beta d_{\mathrm{p}}}\right. \\
& \left.\times\left\{1-\exp \left[-\frac{16 \pi^{3} d_{\mathrm{p}}^{2}}{v^{2}}\left(\frac{\Lambda_{\mathrm{b}}}{A}\right)^{-\frac{1}{2}}\right]\right\}\right)^{-1} \mathrm{~d} \Lambda_{\mathrm{b}}
\end{aligned}
$$

which is the needed formula for $\kappa$ in holey silicon in the non-gray model. Note that in the gray model, where the mfp spectral function is a Dirac delta function, Eq. 34 would lead only to an additional constant factor in the third term of the right-hand side of Eq. 31.

Fitting of Eq. 33 to computed data (Fig. 3) reports an excellent agreement of the gray model with simulations. When pores sit on a cubic lattice, one obtains $\beta=4.9 \pm 0.5$ for $\phi=0.028$ and $\beta=7.6 \pm 1.4$ for $\phi_{\mathrm{p}}=0.005$. Instead, for randomly distributed pores, $\beta=6.8 \pm 1.2$ for $\phi_{\mathrm{p}}=0.005$. Also, the nongray (reflective) model (Eq. 36) shows a more-thanfair agreement with computational data. One obtains $A=(4.6 \pm 2.0) \times 10^{50} \mu \mathrm{m} \mathrm{s}^{-4}$ independently of pore distribution and porosity. The fitting process returns $\beta$ values of $12.6 \pm 2.5$ and $51.7 \pm 11.0$ for $\phi_{\mathrm{p}}=0.028$ and 0.005 with ordered pore distributions; and $\beta=46.6 \pm 12.4$ at $\phi_{\mathrm{p}}=0.005$ for randomly distributed pores.

It should be noted that, beyond their agreement with computational data, the two models predict a
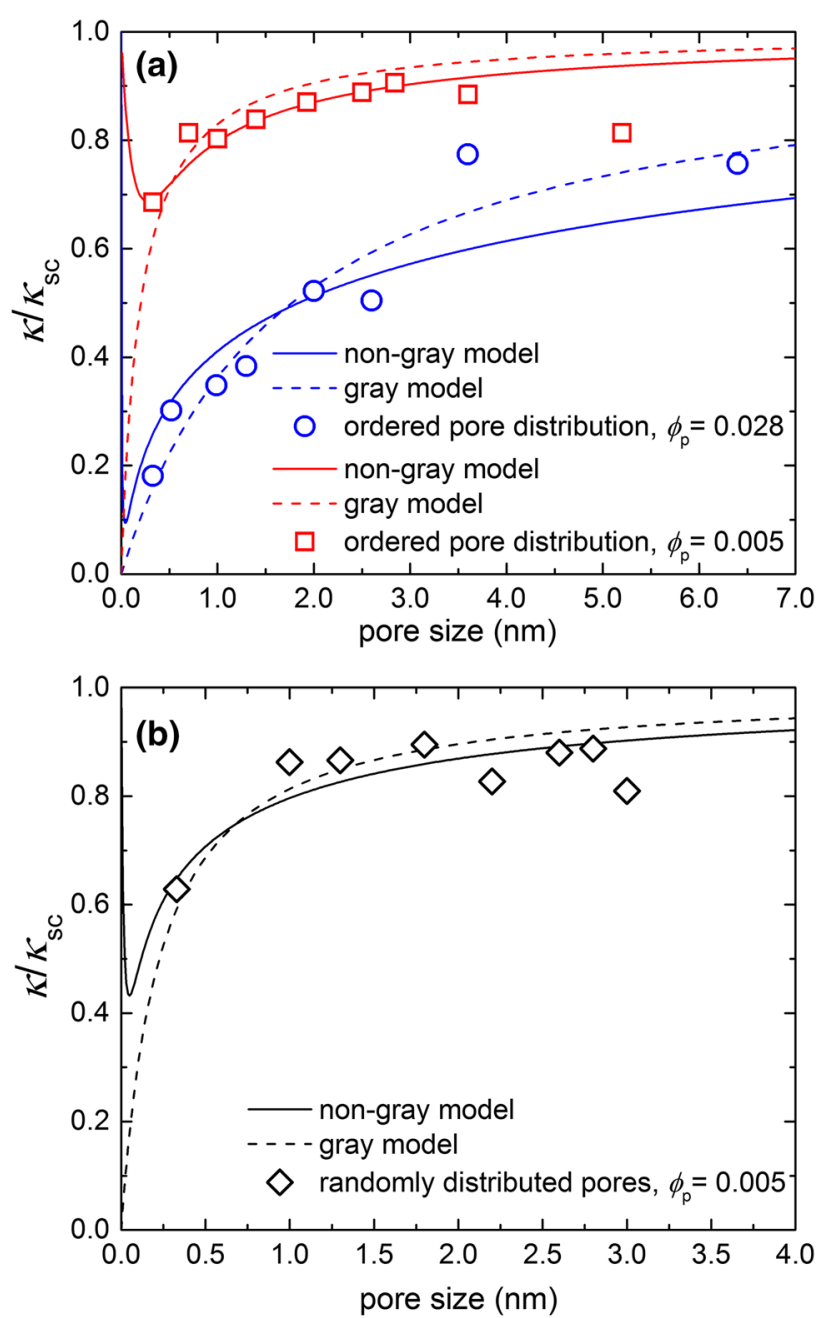

Fig. 3. (color online) Fitting to gray (dashed lines) and non-gray (full lines) scattering models of the normalized values of $\kappa\left(d_{p}\right)$ obtained by simulating (a) an ordered pore distribution and (b) a random distribution of pores in holey single-crystalline silicon. 
completely different behavior of $\kappa\left(d_{\mathrm{p}}\right)$ for $d_{\mathrm{p}} \rightarrow 0$ at constant $\phi_{\mathrm{p}}$. Thermal conductivity in the gray model tends to zero for vanishing pore diameters while it recovers the single-crystal value in the non-gray model, as physical intuition would actually suggest.

\section{Multiple morphological defects in silicon: Holey polycrystalline silicon}

Multiple defects impact thermal conductivity by introducing two classes of scattering bodies in addition to the scattering entities natively present in single-crystalline media. In the gray model, one may use the integral Matthiessen's rule, immediately obtaining from Eqs. 15 and 31 that

$$
\kappa_{\mathrm{HP}, \mathrm{G}}=\kappa_{\mathrm{b}} \frac{2 \beta \gamma_{\mathrm{G}} d_{\mathrm{p}} D}{\gamma_{\mathrm{G}} D\left[\beta d_{\mathrm{p}}(\phi+2)+3 \Lambda_{\mathrm{b}} \phi_{\mathrm{p}}\right]+2 d_{\mathrm{p}} \Lambda_{\mathrm{b}}}
$$

Instead, in the non-gray model, the spectral Matthiessen's rule leads to ${ }^{\dagger}$

$$
\begin{aligned}
\kappa_{\mathrm{HP}, \mathrm{NG}}= & \int_{0}^{\infty} K_{\mathrm{b}}\left(\Lambda_{\mathrm{b}}\right)\left\{1+\frac{\phi_{\mathrm{p}}}{2}+\frac{3 \phi_{\mathrm{p}} \Lambda_{\mathrm{b}}}{2 \beta d_{\mathrm{p}}}\right. \\
& \times\left[1-\exp \left(-\frac{16 \pi^{3} d_{\mathrm{p}}^{2}}{v^{2}}\left(\frac{\Lambda_{\mathrm{b}}}{A}\right)^{-\frac{1}{2}}\right)\right] \\
& \left.+\frac{\gamma \Lambda_{\mathrm{b}}}{\beta \omega_{\max } D}\left(\frac{\Lambda_{\mathrm{b}}}{A}\right)^{-\frac{1}{4}}\right\}^{-1} \mathrm{~d} \Lambda_{\mathrm{b}}
\end{aligned}
$$

Figure 4 directly compares the experimental and computed thermal conductivities, both normalized to the thermal conductivity of single-crystalline Si. A value of $\kappa_{\mathrm{sc}}=120 \mathrm{~W} \mathrm{~m}^{-1} \mathrm{~K}^{-1}$ was used, which is appropriate for sub-micrometric thin films. ${ }^{55}$ For computer simulations, a clearly better agreement is found using the non-gray model, reporting $\beta=30.0 \pm 0.1$ and $27.4 \pm 4.2$ for pores at GBs only or randomly distributed, respectively, and values of $\gamma=4 \pm 1$ and $A=(3.00 \pm 0.99) \times 10^{51} \mu \mathrm{m} \mathrm{s}^{-4}$ in both cases. The gray model returns instead $\beta=1.85 \pm 0.59$ and $\gamma_{\mathrm{G}}=1.00 \pm 0.36$ when pores sit at GBs only; and $\beta=1.90 \pm 0.51$ and $\gamma_{\mathrm{G}}=1.00 \pm 0.31$ for randomly distributed pores.

Experimental data from Dunham et al. ${ }^{17}$ on $\mathrm{He}^{+}$implanted nanocrystalline thin films ${ }^{56,57}$ are fitted instead with $\beta=1.0 \pm 0.5$ and $\gamma_{\mathrm{G}}=0.5 \pm 0.3$ in the gray model, and with $\beta=1.0 \pm 0.6, \gamma=7.0 \pm 3.9$, and $A=(2.29 \pm 1.31) \times 10^{46} \mu \mathrm{m} \mathrm{s}^{-4}$ in the non-gray model. Note that, since the experimental thermal conductivity was measured cross-plane, grain size used in the fitting referred to the grain height, namely $D=150 \mathrm{~nm}$. It should also be remarked how

\footnotetext{
${ }^{\dagger}$ In Eq. 38, we used the same $\beta$ for both scattering mechanisms as the effectiveness of a specific type of scattering event is already accounted for by $R$ for the pores and by $\gamma / \omega_{\max }$ for the grains.
}
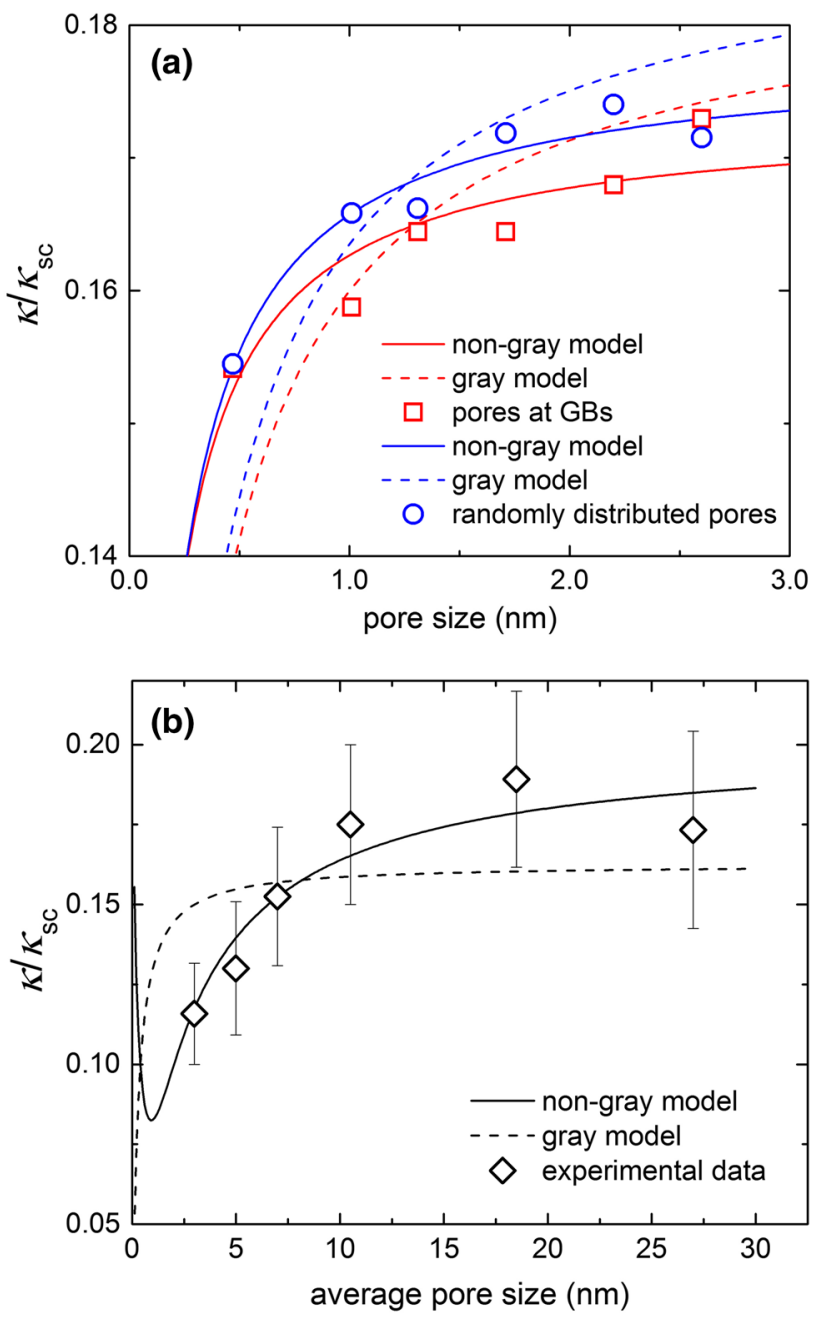

Fig. 4. (color online) (a) Fitting of gray (dashed lines) and non-gray (full lines) scattering models to normalized values of $\kappa\left(d_{p}\right)$ obtained by simulating random distributions of pores in holey polycrystalline silicon. (b) Fitting of gray (dashed lines) and non-gray (full lines) scattering models to normalized values of $\kappa\left(d_{\mathrm{p}}\right)$ measured in $\mathrm{He}^{+}-$ implanted nanocrystalline silicon. Experimental data from Ref. 17.

the gray model predicts an almost constant $\kappa$ for $d>5 \mathrm{~nm}$, in good agreement with with gray Monte Carlo simulations reported previously. ${ }^{17}$

\section{DISCUSSION}

Comparison of fits to both experimental and computed data suggests that non-gray models are more accurate than gray ones. This is not completely obvious, as it is reported that relevant differences between gray and non-gray models should be observed mostly at low temperatures. ${ }^{9}$ Instead, even around room temperature and in the presence of a single $\mathrm{MoD}$, it is evident that, although the gray model may provide an acceptable quantitative prediction of the thermal conductivity, ${ }^{17,35}$ a more precise analysis of morphologically limited thermal conductivity requires relaxing the assumption of a single dominant phonon mode governing heat transport. 
However, in spite of its ability to describe the dependence of $\kappa$ upon the density of the pertinent MoD, the non-gray model also raises questions when applied to holey crystals. Specifically, concerns may be raised about its physical soundness in the description of $\kappa$ dependence on $d_{\mathrm{p}}$ over the whole range of pore sizes. Gray and non-gray models, while mostly showing the same expected decrease of $\kappa$ with decreasing $d_{\mathrm{p}}$, dramatically differ from each other in the zero-size limit. We note that, while in holey single-crystalline silicon this difference occurs at pore sizes comparable to the size of a single vacancy, in holey polycrystalline silicon the departure between the two models shows up instead for $d_{\mathrm{p}} \approx 1 \mathrm{~nm}$, i.e. when pores are well-defined, extended morphological defects. The gray model predicts a vanishing thermal conductivity, while the non-gray model predicts a recovery of $\kappa$ to the porefree value. Such a difference is clearly explained by inspection of Eqs. 32 and 35. In both cases, the mfp is dominated by phonon scattering at pores. However, the gray model considers all scattering events as effective while the non-gray model accounts for the probability that phonons are elastically reflected at pore surfaces. In the latter case, when both $d_{\mathrm{p}}$ and $\ell_{\mathrm{p}}$ tend to zero (as needed to keep $\phi_{\mathrm{p}}$ constant), no collision remains effective, so that the material recovers the conductivity pertaining to the pore-free material. However, both predictions are questionable. In the gray model, the assumption that all collisions are effective (namely that in no case phonons may be elastically reflected at pore surfaces) sensibly overestimates the effectiveness of pores as scattering centers. Conversely, Eq. 34 provides an oversimplified description of the actual reflectivity at internal surfaces ${ }^{36}$ that in turn underestimates scatter efficiency in the low $d_{\mathrm{p}}$ limit. A more fundamental limitation of both models arises when considering non-local effects. Since for $d_{\mathrm{p}} \rightarrow 0$ at fixed $\phi_{\mathrm{p}}$ the pore density abruptly increases, one may expect lattice distortions due to pores to extend over a larger portion of the crystal, a feature that is not accounted for in either gray or non-gray models. At high porosity, this should lead to an increase of positional disorder, up to a quasiamorphization of the solid. Thus, $\kappa$ may be expected to approach the Casimir limit. At low porosity, instead, pores will degenerate into a collection of sparsely distributed vacancies, imparting a negligible increase of lattice disordering and simply increasing the vacancy density. Thus, $\kappa$ should almost recover its pore-free value: neither gray nor non-gray models are adequate at accounting for the effect of pores at any porosity, as neither of them properly encompasses non-local effects due to porerelated lattice disorder. Non-gray models possibly better qualify in the current case because of the relatively small $\phi_{\mathrm{p}}$ considered in this study. One would then draw the conclusion that while the nongray model is a more appropriate choice even at non-cryogenic temperatures, it is the use of Matthiessen's rule (either integral or spectral) which determines the limits of applicability for either model. We have shown that MoD-related scattering lengths $\left(\ell_{\mathrm{p}}\right.$ and $\left.\gamma_{\mathrm{G}} D\right)$ sensibly depend not only on the distribution of scattering centers within the medium but also on the medium itself. Thus, non-local descriptors accounting for the lattice distortion around MoDs are needed.

Finally, concerning the usability of more MoDs to tailor the thermal conductivity, we found that the addition of a morphological defect to an already defective material causes a further decrease of its thermal conductivity if the two sets of defects have different characteristic sizes. Experimental results show the conductivity to drop by about $30 \%$, with smaller decreases $(\approx 10 \%)$ being reported by simulations. We may then conclude that the use of a variety of MoDs to control a material's thermal conductivity may be safely planned by the use of non-gray models and of the spectral Matthiessen's rule. This result is consistent with the conclusions of previous publications ${ }^{17,56,57}$ and further stresses how the introduction of pores in heavily borondoped nanocrystalline silicon showing enhanced power factors ${ }^{28,29,58}$ may lead to large thermoelectric efficiencies in a fairly geo-abundant material.

\section{SUMMARY AND CONCLUSIONS}

In this paper, we analyzed the effect of morphological defects on the thermal conductivity of silicon. We found that further reduction of the thermal conductivity may be achieved by introducing more sets of defects with different characteristic sizes. This is a relevant point as heavily doped nanocrystalline silicon has been reported to display large thermoelectric power factors, so that additional reduction of its thermal conductivity might impart to it very large thermoelectric figures of merit in excess of one even around room temperature.

Using the Yang-Dames formalism, we have also shown how $\kappa$ can be effectively modelled using the spectral Matthiessen's rule, thus providing an efficient tool to guide defect engineering. However, physical inconsistencies of the model were singled out in the range of very low pore sizes, suggesting that changes in the structure of the $B_{\mathrm{b}}^{\mathrm{MoD}}\left(\Lambda_{\mathrm{b}}, \ell_{\mathrm{MoD}}\right)$ are needed beyond Matthiessen's rule to more properly account for non-local effects related to lattice disordering induced by pores over a wider range of porosities.

\section{ACKNOWLEDGEMENTS}

B.L., D.N., and R.T. are grateful to Prof. Giampiero Ottaviani for his continual support throughout this work and the many useful discussions. B.L. and D.N. acknowledge the partial support received by FP7-NMP-2013-SMALL-7 in the frame of Silicon Friendly Materials and Device Solutions for Microenergy Applications (SiNERGY) Project, Con- 
tract No. 604169. L.C. acknowledges financial support by Regione Sardegna under the Fundamental Research Project No. CRP78744 'Energy applications with porous silicon (ENAPSi)'. R.D. acknowledges Regione Sardegna for financial support under project P.O.R. Sardegna F.S.E. 2007-2013 (Axis IV Human Resources, Objective 1.3, Line of Activity 1.3.1). Computational support by CINECA under project ISCRA-TREWI is acknowledged as well. M.T.D. acknowledges ASEE for funding through the National Defense Science and Engineering Graduate fellowship.

\section{REFERENCES}

1. H.P. Li, A.D. Sarkar, and R.Q. Zhang, Europhys. Lett. 96, 56007 (2011).

2. J. Chen, G. Zhang, and B. Li, Nano Lett. 12, 2826 (2012).

3. G. Zhang and B. Li, Nanoscale 2, 1058 (2010).

4. A.I. Hochbaum, R.K. Chen, R.D. Delgado, W.J. Liang, E.C. Garnett, M. Najarian, A. Majumdar, and P.D. Yang, $\mathrm{Na}$ ture 451, 163 (2008).

5. A.I. Boukai, Y. Bunimovich, J. Tahir-Kheli, J.K. Yu, W.A. Goddard, and J.R. Heath, Nature 451, 168 (2008).

6. D. Song and G. Chen, Appl. Phys. Lett. 84, 687 (2004).

7. F. Yang and C. Dames, Phys. Rev. B 87, 035437 (2013).

8. M. Nomura, Y. Kage, D. Müller, D. Moser, and O. Paul, Appl. Phys. Lett. 106, 223106 (2015).

9. Z. Wang, J.E. Alaniz, W. Jang, J.E. Garay, and C. Dames, Nano Lett. 11, 2206 (2011).

10. C. Hua and A.J. Minnich, Semicond. Sci. Technol. 29, 124004 (2014).

11. J. Ma, B.R. Parajuli, M.G. Ghossoub, A. Mihi, J. Sadhu, P.V. Braun, and S. Sinha, Nano Lett. 13, 618 (2013).

12. J. Fang, C.B. Kang, Y. Huang, S.H. Tolbert, and L. Pilon, J. Phys. Chem. C 116, 12926 (2012).

13. D.G. Cahill and R. Pohl, Annu. Rev. Phys. Chem. 39, 93 (1988).

14. P. Jiang, L. Lindsay, and Y.K. Koh, J. Appl. Phys. 119, 245705 (2016).

15. L.D. Zhao, V.P. Dravid, and M.G. Kanatzidis, Energy Environ. Sci. 7, 251 (2014).

16. G. Zhang and Y.W. Zhang, Phys. Status Solidi RRL 7, 754 (2013).

17. M. Dunham, B. Lorenzi, S. Andrews, A. Sood, M. Asheghi, D. Narducci, and K. Goodson, Appl. Phys. Lett. 109, 253105 (2016).

18. M. Asheghi, M. Touzelbaev, K. Goodson, Y. Leung, and S. Wong, J. Heat Transf. 120, 30 (1998).

19. Y.S. Ju and K.E. Goodson, Appl. Phys. Lett. 74, 3005 (1999).

20. W. Liu and M. Asheghi, J. Appl. Phys. 98, 123523 (2005).

21. D. Narducci, G. Cerofolini, M. Ferri, F. Suriano, F. Mancarella, L. Belsito, S. Solmi, and A. Roncaglia, J. Mater. Sci. 48, 2779 (2013).

22. Z. Aksamija and I. Knezevic, Phys. Rev. B 82, 045319 (2010).

23. L. Weber and E. Gmelin, Appl. Phys. A 53, 136 (1991).

24. S.K. Bux, R.G. Blair, P.K. Gogna, H. Lee, G. Chen, M.S. Dresselhaus, R.B. Kaner, and J.P. Fleurial, Adv. Funct. Mater. 19, 2445 (2009).

25. T. Claudio, N. Stein, D.G. Stroppa, B. Klobes, M.M. Koza, P. Kudejova, N. Petermann, H. Wiggers, G. Schierning, and R.P. Hermann, Phys. Chem. Chem. Phys. 16, 25701 (2014).
26. Z. Aksamija, J. Electron. Mater. 44, 1644 (2015).

27. N. Neophytou, X. Zianni, H. Kosina, S. Frabboni, B. Lorenzi, and D. Narducci, Nanotechnology 24, 205402 (2013).

28. D. Narducci, B. Lorenzi, X. Zianni, N. Neophytou, S. Frabboni, G.C. Gazzadi, A. Roncaglia, and F. Suriano, Phys. Status Solidi A 211, 1255 (2014).

29. D. Narducci, S. Frabboni, and X. Zianni, J. Mater. Chem. C 3, 12176 (2015).

30. N.S. Bennett, N.M. Wight, S.R. Popuri, and J.W.G. Bos, Nano Energy 16, 350 (2015).

31. L. Yang, J. Wu, and L. Zhang, J. Alloys Compd. 364, 83 (2004).

32. A.U. Khan, K. Kobayashi, D.M. Tang, Y. Yamauchi, K. Hasegawa, M. Mitome, Y. Xue, B. Jiang, K. Tsuchiya, D. Golberg, Y. Bando, and T. Mori, Nano Energy 31, 152 (2017).

33. M. Luisier, Appl. Phys. Lett. 103, 113103 (2013).

34. T. Feng, B. Qiu, and X. Ruan, Phys. Rev. B 92, 235206 (2015).

35. X. Cartoixà, R. Dettori, C. Melis, L. Colombo, and R. Rurali, Appl. Phys. Lett. 109, 013107 (2016).

36. J. Ziman, Electrons and Phonons: The Theory of Transport Phenomena in Solids. Oxford Classic Texts in the Physical Sciences (OUP Oxford, Oxford, 2001).

37. K. Esfarjani, G. Chen, and H.T. Stokes, Phys. Rev. B 84, 085204 (2011).

38. R. Dettori, C. Melis, X. Cartoixà, R. Rurali, and L. Colombo, Phys. Rev. B 91, 054305 (2015).

39. L. Brambilla, L. Colombo, V. Rosato, and F. Cleri, Appl. Phys. Lett. 77, 5 (2000).

40. C. Melis and L. Colombo, Phys. Rev. Lett. 112, 5 (2014).

41. F.J. Justo, Z.M. Bazant, E. Kaxiras, V.V. Bulatov, and S. Yip, Phys. Rev. B 58, 2539 (1998).

42. C. Melis, R. Dettori, S. Vandermeulen, and L. Colombo, Eur. Phys. J. B 87, 96 (2014).

43. R. Dettori, C. Melis, and L. Colombo, Eur. Phys. J. B 88, 27 (2015).

44. S. Plimpton, J. Comput. Phys. 117, 1 (1995).

45. G. Barbarino, C. Melis, and L. Colombo, Phys. Rev. B 91, 035416 (2015).

46. K.R. Hahn, C. Melis, and L. Colombo, J. Phys. Chem. C 120, 3026 (2016).

47. S. Das Sarma and F. Stern, Phys. Rev. B 32, 8442 (1985).

48. J. Yang and T. Caillat, MRS Bull. 31, 224 (2006).

49. J. Callaway, Phys. Rev. 113, 1046 (1959).

50. J.J. Moré, Numerical Analysis (Berlin: Springer, 1978), pp. $105-116$

51. L. Ingber, Math. Comput. Model. 18, 29 (1993).

52. R. Tubino, L. Piseri, and G. Zerbi, J. Chem. Phys. 56, 1022 (1972).

53. S. Torquato, Random Heterogeneous Materials: Microstructure and Macroscopic Properties. Interdisciplinary Applied Mathematics (Springer, New York, 2005).

54. V. Chiloyan, J. Garg, K. Esfarjani, and G. Chen, Nat. Commun. 6, 6755 (2015).

55. M. Asheghi, K. Kurabayashi, R. Kasnavi, and K.E. Goodson, J. Appl. Phys. 91, 5079 (2002).

56. B. Lorenzi, D. Narducci, R. Tonini, S. Frabboni, G.C. Gazzadi, G. Ottaviani, N. Neophytou, and X. Zianni, J. Electron. Mater. 43, 3812 (2014).

57. B. Lorenzi, S. Frabboni, G.C. Gazzadi, R. Tonini, G. Ottaviani, and D. Narducci, J. Electron. Mater. 43, 3852 (2014).

58. D. Narducci, E. Selezneva, G. Cerofolini, S. Frabboni, and G. Ottaviani, J. Solid State Chem. 193, 19 (2012). 